\title{
Comparison of Coupling Intervals That Induce Clinical and Nonclinical Forms of Ventricular Tachycardia During Programmed Stimulation
}

\author{
FRED MORADY, MD, LORENZO A. DICARLO, Jr., MD, \\ JEFFREY M. BAERMAN, MD, and MICHAEL de BUITLEIR, MB
}

Coupling intervals of extrastimuli that induced 57 previously documented unimorphic ventricular tachycardias (VTs) were compared with coupling intervals that induced 57 episodes of polymorphic VT or ventricular fibrillation (VF) in patients without a documented or suspected history of polymorphic VT or VF. Programmed stimulation was performed with the patient in the drug-free state, with 1 to 3 extrastimuli and 2 basic drive cycle lengths $(600$ or $500 \mathrm{~ms}$, and $400 \mathrm{~ms}$ ) at 2 right ventricular sites; stimuli were twice diastolic threshold. The mean coupling intervals of the first, second and third extrastimuli that induced nonclinical VT/VF (241 \pm $19,185 \pm 19$ and $173 \pm 24 \mathrm{~ms}$, respectively, mean \pm standard deviation) were significantly shorter than the corresponding coupling intervals that induced the clinical VTs $(266 \pm 25,228 \pm 32$ and $214 \pm 27 \mathrm{~ms}$, respectively, $p<0.001$ for each). Regardless of the basic drive cycle length, the shortest coupling interval required to induce a clinical VT was $180 \mathrm{~ms}$. Depending on the drive cycle length, 29 to $70 \%$ of nonclinical VT/VF induced by 3 extrastimuli required a coupling interval of less than $180 \mathrm{~ms}$ to induce. Therefore, a lower limit of coupling intervals may be identified below which only nonclinical VT/VF is induced by programmed stimulation. Restriction of coupling intervals to this lower limit may allow for significant improvement in specificity without compromise in the sensitivity of programmed ventricular stimulation protocols.

(Am J Cardiol 1986;57:1269-1273) nimorphic ventricular tachycardia (VT) that occurs spontaneously can often be induced by programmed ventricular stimulation. The yield of clinically significant forms of VT progressively increases as the number of extrastimuli in the stimulation protocol increases. ${ }^{1-5}$ However, programmed ventricular stimulation may also induce ventricular fibrillation (VF) or rapid, polymorphic VT, which often represent laboratory artifacts of no clinical significance. ${ }^{6}$ The yield of these nonclinical arrhythmias also increases as the number of extrastimuli used during programmed stim-

From the Division of Cardiology, Department of Internal Medicine, University Hospitals, University of Michigan Medical Center, Ann Arbor, Michigan. Manuscript received December 6, 1985; revised manuscript received January 21, 1986, accepted January 22, 1986.

Address for reprints: Fred Morady, MD, Division of Cardiology, University Hospital, 1500 East Medical Center Drive, Ann Arbor, Michigan 48109 . ulation increases. ${ }^{1-4,7,8}$ Because nonclinical forms of polymorphic VT or VF often require countershock to terminate, it is desirable to minimize the induction of these arrhythmias.

Coupling intervals that induce polymorphic VT or VF may be shorter than the coupling intervals that induce unimorphic VT. ${ }^{9}$ It is therefore possible that a limitation of coupling intervals without a decrease in the number of extrastimuli might improve the specificity of programmed ventricular stimulation without impairing sensitivity. However, no studies have critically analyzed or compared the coupling intervals that induce clinical and nonclinical forms of VT/VF. This study compares the coupling intervals that induced clinical forms of unimorphic VT and nonclinical forms of polymorphic VT or VF, using a stimulation protocol that includes up to 3 ventricular extrastimuli. Our aim was to evaluate whether there is a lower limit of coupling intervals below which only nonclinical forms of VT are induced. 


\begin{tabular}{|c|c|c|}
\hline & Clinical VTs & Nonclinical VTs \\
\hline n & 57 & 57 \\
\hline Cycle length (ms) (mean \pm SD) & $309 \pm 60$ & $192 \pm 18^{*}$ \\
\hline \multicolumn{3}{|l|}{ Duration } \\
\hline Nonsustained & 0 & 29 \\
\hline Sustained & 57 & 28 \\
\hline \multicolumn{3}{|l|}{ Configuration of VT } \\
\hline Right bundle branch block & 39 & 0 \\
\hline Left bundle branch block & 18 & 0 \\
\hline Polymorphic & 0 & 44 \\
\hline Ventricular fibrillation & 0 & 13 \\
\hline \multicolumn{3}{|l|}{ Induction technique } \\
\hline One extrastimulus & $10(18 \%)$ & 0 \\
\hline Two extrastimuli & $31(54 \%)$ & $14(24 \%)$ \\
\hline Three extrastimuli & $16(28 \%)$ & $43(7.6 \%)$ \\
\hline
\end{tabular}

* $p<0.001$ vs cycle length of clinical VT.

$\mathrm{SD}=$ standard deviation; $\mathrm{VT}=$ ventricular tachycardia

\section{Methods}

Definitions: VT induced by programmed ventricular stimulation was categorized as clinical if it was unimorphic and similar in configuration to a patient's spontaneous episode of VT, as determined by a 12lead electrocardiogram.

A ventricular arrhythmia induced by programmed ventricular stimulation was categorized as nonclinical if it was polymorphic VT or VF and if it was induced in a patient who did not have a history of polymorphic VT or VF. All patients had had at least 24 hours of continuous electrocardiographic monitoring demonstrating the absence of polymorphic VT, and no patient had a history of out-of-hospital cardiac arrest or VF. The nonclinical polymorphic VT/VFs included in this study were induced in patients with documented sustained or nonsustained unimorphic VT, supraventricular tachycardia, sick sinus syndrome, carotid hypersensitivity or atrioventricular conduction disturbances who underwent programmed ventricular stimulation in the course of a complete electrophysiologic study, or in patients with unexplained syncope. Polymorphic VT and VF induced in patients with unexplained syncope do not have clinical significance. ${ }^{6,10-12}$ However, polymorphic VT or VF induced in patients with a history of cardiac arrest was not included in this study, because the clinical significance of polymorphic VT or $\mathrm{VF}$ in such patients is unclear. ${ }^{6}$

Sustained VT was defined as VT at least 30 seconds in duration or that required countershock for termination. Nonsustained VT was defined as VT at least 6 beats in duration that terminated spontaneously within 30 seconds.

Clinical ventricular tachycardias: Fifty-seven clinical VTs were induced in 52 patients who had a history of documented, sustained, unimorphic VT. The characteristics of these VTs are described in Table I. The patients in whom these VTs were induced consisted of 44 men and 9 women, mean age $57 \pm 12$ years $[ \pm$ standard deviation). Forty-three patients had coronary artery disease (with a history of myocardial infarction in 36], 2 idiopathic dilated cardiomyopathy, 2 mitral valve prolapse and 5 no identifiable structural heart disease.

Nonclinical ventricular tachycardias: Nonclinical polymorphic VT or VF was induced in 57 patients. The characteristics of these episodes of induced VT or VF are described in Table I. Patients in whom these tachy. cardias were induced consisted of 37 men and 20 women, mean age $62 \pm 9$ years. Thirty-five patients had coronary artery disease (with a history of myocardial infarction in 28), 10 idiopathic dilated cardiomyopathy, 3 mitral valve prolapse and 9 no identifiable structural heart disease.

Electrophysiologic study protocol: Patients underwent electrophysiologic study in the fasting, unsedated state after they gave informed consent. All studies were performed at least 4 half-lives after all antiarrhythmic drug treatment had been discontinued. Two quadripolar electrode catheters were inserted percutaneously into a femoral vein. Whenever indicated, atrial stimulation was performed; then the catheters were positioned against the apex of the right ventricle and the right ventricular outflow tract or septum. Electrocardiographic leads $\mathrm{V}_{1}$, I and III, and the intracardiac electrograms were displayed on an oscilloscope and recorded on an Electronics for Medicine VR 16 recorder at a paper speed of $25 \mathrm{~mm} / \mathrm{s}$. In addition, whenever sustained unimorphic VT was induced, a 12-lead electrocardiogram was recorded. Stimulation was performed using a programmable stimulator (Bloom Associates, Ltd.) with stimuli that were $2 \mathrm{~ms}$ in duration and twice diastolic threshold. In all patients, the diastolic threshold was $0.8 \mathrm{~mA}$ or less.

Programmed stimulation was performed using 6- to 8-beat drive trains at 2 basic drive cycle lengths, either 600 or $500 \mathrm{~ms}$, and $400 \mathrm{~ms}$. Coupling intervals of the first, second and third extrastimuli were designated as $\mathrm{S}_{1} \mathrm{~S}_{2}, \mathrm{~S}_{2} \mathrm{~S}_{3}$ and $\mathrm{S}_{3} \mathrm{~S}_{4}$, respectively. Programmed stimulation was initiated at the right ventricular apex with an $S_{1} S_{2}$ of 350 to $450 \mathrm{~ms}$, depending on the basic drive cycle length. Coupling intervals were decreased in steps of $10 \mathrm{~ms}$. Programmed stimulation with double extrastimuli was performed starting with an $S_{1} S_{2} 30 \mathrm{~ms}$ beyond the effective refractory period and with an $S_{2} S_{3}$ of $300 \mathrm{~ms}$. When the second extrastimulus $\left(S_{3}\right)$ reached refractoriness, $S_{1} S_{2}$ was decreased by $10-\mathrm{ms}$ steps until $S_{3}$ again evoked a response. $S_{2} S_{3}$ was then decreased until $S_{3}$ again reached refractoriness, and $\mathrm{S}_{1} \mathrm{~S}_{2}$ was again decreased by $10 \mathrm{~ms}$ steps until $\mathrm{S}_{3}$ again evoked a response. This process was continued until the first extrastimulus $\left(\mathrm{S}_{2}\right)$ no longer evoked a response. After programmed stimulation at 2 basic drive cycle lengths with 1 and 2 extrastimuli was completed at the right ventricular apex, these same steps were performed at the second right ventricular site. Programmed ventricular stimulation with 3 extrastimuli was then performed at the right ventricular apex, with $S_{2}$ and $S_{3}$ positioned $30 \mathrm{~ms}$ beyond their respective points of refractoriness, and with an initial $\mathrm{S}_{3} \mathrm{~S}_{4}$ of $300 \mathrm{~ms}$. When the third extrastimulus $\left(\mathrm{S}_{4}\right)$ no longer evoked a response, the $S_{2} S_{3}$ interval was decreased in 
TABLE II Comparison of the Mean Coupling Intervals that Induced 57 Eplsodes of Clinical Ventricular Tachycardia and 57 Episodes of Nonclinical Ventricular Tachycardia or Ventricular Ellbrt!lation

\begin{tabular}{llcl}
\hline & Clinical VTs & Nonclinical VT/VFs & p Value \\
\hline$S_{1} S_{2}(m s)$ & $266 \pm 25^{*}$ & $241 \pm 19$ & $<0.001$ \\
$S_{2} S_{3}(m s)$ & $228 \pm 32$ & $185 \pm 19$ & $<0.001$ \\
$S_{3} S_{4}(m s)$ & $214 \pm 27$ & $173 \pm 24$ & $<0.001$ \\
\hline
\end{tabular}

"Mean \pm standard deviation.

$S_{1} S_{2}, S_{2} S_{3}$ and $S_{3} S_{4}=$ coupling intervals of first, second and third extrastimuli, respectively; VF $=$ ventricular fibrillation; $V T=$ ventricular tachycardia.

10-ms steps until $\mathrm{S}_{4}$ again evoked a response. This process was continued until $S_{3}$ no longer evoked a response, at which point the $\mathrm{S}_{1} \mathrm{~S}_{2}$ interval was decreased by 10 -ms steps until $S_{3}$ again evoked a response. This was continued until $S_{2}$ no longer evoked a response. After programmed stimulation with 3 extrastimuli at 2 basic drive cycle lengths at the right ventricular apex, 3 extrastimuli were then introduced at the second right ventricular site.

The endpoint of the stimulation protocol in patients who had a history of VT was the induction of each patient's clinical VT. In patients without a history of VT, the endpoint was induction of VT requiring countershock for termination. Reproducibility of an induced VT was assessed except when cuuntershock was necessary to terminate the VT. In many patients VT was reproducibly inducible, but the coupling intervals that induced the VT were variable. In these cases, the coupling intervals that induced the first episode of VT were noted.

Statistical analysis: Statistical comparisons were performed with Student $t$ test or by analysis of variance. A $\mathrm{p}$ value $<0.05$ was considered significant.

\section{Results}

The mean $S_{1} S_{2}, S_{2} S_{3}$ and $S_{3} S_{4}$ intervals that induced the clinical and nonclinical VTs are listed in Table II. The mean $\mathbf{S}_{1} \mathbf{S}_{2}, \mathbf{S}_{2} \mathbf{S}_{3}$ and $\mathbf{S}_{3} \mathbf{S}_{4}$ intervals that induced the nonclinical VTs were each significantly shorter than the corresponding mean coupling intervals of the extrastimuli that induced the clinical VTs $(p<0.001)$.

Among the clinical VTs there was no significant difference in the mean of the coupling intervals that induced VT at a basic drive cycle length of 600,500 or $400 \mathrm{~ms}$. Among the nonclinical VTs, mean coupling intervals of the extrastimuli that induced VT/VF at a basic drive cycle length of 600 and $500 \mathrm{~ms}$ were not significantly different. However, at a basic drive cycle length of $400 \mathrm{~ms}$, the mean coupling intervals that resulted in the induction of nonclinical VT or VF were significantly shorter than the corresponding coupling intervals at a basic drive cycle length of 600 and $500 \mathrm{~ms}$ (Table III). There was no significant difference in the mean $S_{1} S_{2}, S_{2} S_{3}$ or $S_{3} S_{4}$ intervals that induced the 29 episodes of nonsustained nonclinical VT and the 28 episodes of sustained nonclinical VT or VF.

The actual coupling intervals that induced the 114 episodes of VT/VF are shown in Figure 1. The shortest
TABLE III Comparison of Mean Coupling Intervals that Induced Nonclinical Ventricular Tachycardia or Ventricular Fibrillation at a Basic Drive Cycle Length of 600 and $500 \mathrm{~ms}$ vs $400 \mathrm{~ms}$

\begin{tabular}{|c|c|c|c|}
\hline & \multicolumn{2}{|c|}{ 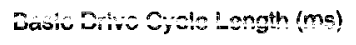 } & \multirow[b]{2}{*}{ p Value } \\
\hline & $600 / 500^{*}$ & 400 & \\
\hline $\mathrm{s}_{1} \mathrm{~s}_{2}(\mathrm{~ms})$ & $248 \pm 17^{\dagger}$ & $233 \pm 18$ & $<0.01$ \\
\hline $\mathrm{S}_{2} \mathrm{~S}_{3}(\mathrm{~ms})$ & $194 \pm 19$ & $175 \pm 16$ & $<0.001$ \\
\hline $\mathrm{S}_{2} \mathrm{~S}_{4}(\mathrm{~ms})$ & $182 \pm 24$ & $162 \pm 19$ & $<0.01$ \\
\hline
\end{tabular}

* Mean coupling intervals at a basic drive cycle length of 600 and $500 \mathrm{~ms}$ were not significantly different and have been pooled.

$\dagger$ Values are mean \pm standard deviation.

Abbreviations as in Table II.

$\mathrm{S}_{2} \mathrm{~S}_{3}$ or $\mathrm{S}_{3} \mathrm{~S}_{4}$ interval required to induce a clinical VT was $180 \mathrm{~ms}$. Among the 14 episodes of nonclinical VT or VF induced by 2 extrastimuli, $2(14 \%)$ were induced by an $S_{2} S_{3}$ interval of less than $180 \mathrm{~ms}$. Among the 43 episodes of nonclinical VT or VF induced by 3 extrastimuli, $23(53 \%)$ were induced by an $\mathrm{S}_{2} \mathrm{~S}_{3}$ or $\mathrm{S}_{3} \mathrm{~S}_{4}$ interval of less than $180 \mathrm{~ms}$. At a basic drive cycle length of 600 or $500 \mathrm{~ms}, 23$ episodes of nonclinical VT

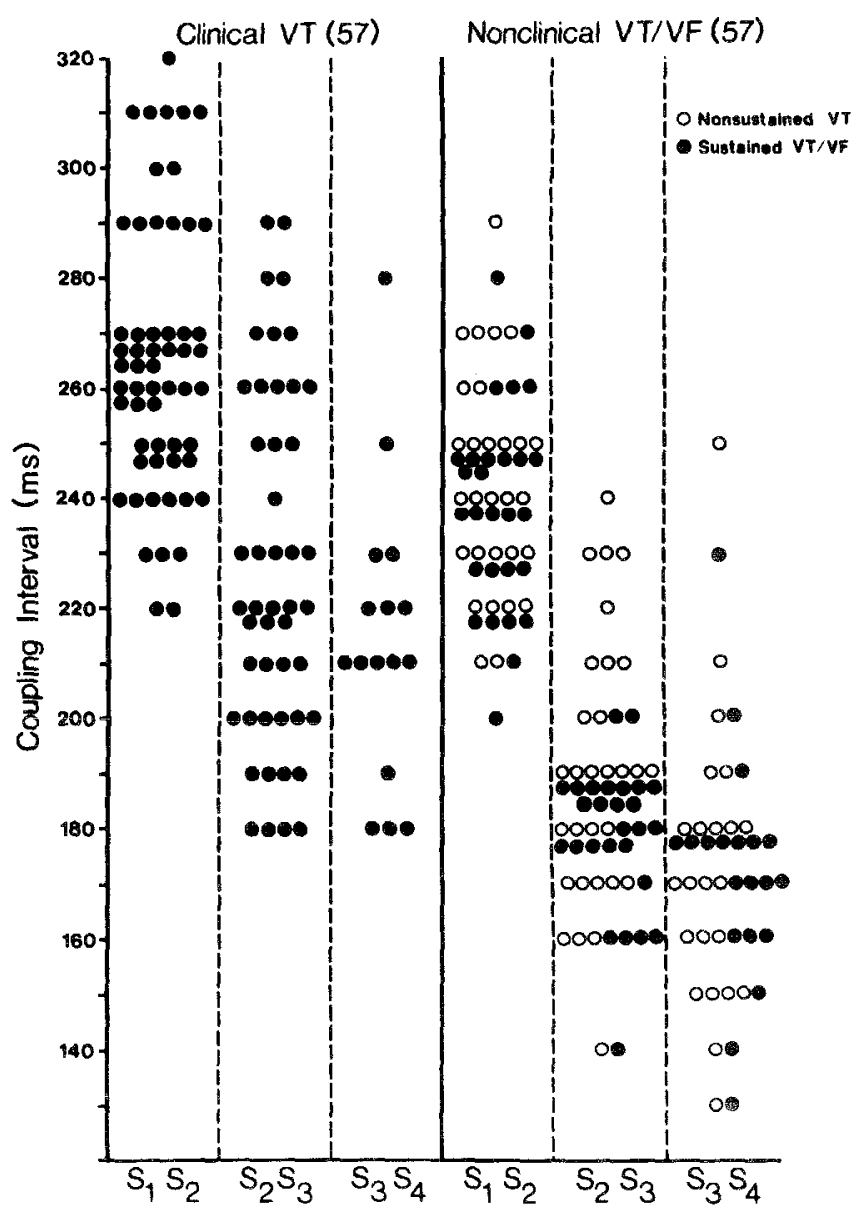

FIGURE 1. Coupling Intervals that induced 57 eplsodes of clinlcal ventricular tachycardla (VT) and 57 episodes of nonclinical VT or ventricular fibrllation (VF). Coupling intervals at basic drive cycle lengths of 600,500 and $400 \mathrm{~ms}$ have been pooled. $s_{1} s_{2}, s_{2} s_{3}$ and $S_{3} S_{4}=$ coupling intervals of first, second and third extrastimull, respectively. 
or VF were induced by 3 extrastimuli, of which $9(39 \%)$ required an $\mathrm{S}_{2} \mathrm{~S}_{3}$ and/or $\mathrm{S}_{3} \mathrm{~S}_{4}$ interval of less than 180 $\mathrm{ms}$. At a basic drive cycle length of $400 \mathrm{~ms}, 20$ episodes of nonclinical VT or VF were induced by triple extrastimuli, of which $14(70 \%)$ required an $\mathrm{S}_{2} \mathrm{~S}_{3}$ or $\mathrm{S}_{3} \mathrm{~S}_{4}$ interval of less than $180 \mathrm{~ms}$.

\section{Discussion}

The results of this study demonstrate that the coupling intervals required to induce nonclinical forms of VT or VF are often shorter than the coupling intervals required to induce clinical VTs during programmed ventricular stimulation. The shortest $\mathrm{S}_{2} \mathrm{~S}_{3}$ or $\mathrm{S}_{3} \mathrm{~S}_{4}$ coupling interval that resulted in the induction of clinical VT was $180 \mathrm{~ms}$, whereas a large proportion of nonclinical forms of VT or VF (up to 70\% at a basic drive cycle length of $400 \mathrm{~ms}$ ) were induced with an $\mathrm{S}_{2} \mathrm{~S}_{3}$ or $\mathrm{S}_{3} \mathrm{~S}_{4}$ interval of less than $180 \mathrm{~ms}$.

These results have important implications regarding the sensitivity and specificity of programmed ventricular stimulation protocols. The yield of nonclinical VT or VF may be significantly decreased without compromising the yield of clinical VT by use of a programmed ventricular stimulation protocol in which the $S_{2} S_{3}$ and $S_{3} S_{4}$ intervals are limited to $180 \mathrm{~ms}$ each (at basic drive cycle lengths of 600,500 and $400 \mathrm{~ms}$ ).

In this study, $28 \%$ of clinical VTs required 3 extrastimuli to induce. Simtlarly, previous studies have reported that the use of 3 extrastimuli during programmed ventricular stimulation increases the yield of clinical VT by approximately $25 \% .1^{1-4}$ However, in the present and in previous studies, the use of 3 extrastimuli was responsible for the induction of most of the nonclinical forms of VT or VF that were induced by programmed ventricular stimulation. ${ }^{1-4,7,8}$ Therefore, while needed to induce a significant proportion of clinically relevant VTs, 3 extrastimuli may also result in induction of a clinically irrelevant form of polymorphic VT or VF that may require direct-current countershock to terminate. Our results indicate that limiting the $S_{2} S_{3}$ and $S_{3} S_{4}$ intervals to $180 \mathrm{~ms}$, while not completely eliminating the possibility of inducing a nonclinical arrhythmia with 3 extrastimuli, will significantly decrease the yield of nonclinical VT or VF without decreasing the yield of clinical VTs. The degree by which the yield of nonclinical arrhythmias is diminished is dependent on the basic drive cycle length $39 \%$ at a basic drive cycle length of 600 or 500 $\mathrm{ms}$ and $70 \%$ at a basic drive cycle length of $400 \mathrm{~ms}$ ).

Whereas the concept that specificity of a programmed ventricular stimulation protocol can be improved without compromising sensitivity may be generally applicable, the specific recommendation that the $\mathrm{S}_{2} \mathrm{~S}_{3}$ and $\mathrm{S}_{3} \mathrm{~S}_{4}$ intervals be limited to $180 \mathrm{~ms}$ clearly applies only to stimulation protocols similar to that used in the present study. The minimum $\mathrm{S}_{2} \mathrm{~S}_{3}$ or $\mathrm{S}_{3} \mathrm{~S}_{4}$ intervals needed to induce a clinical form of VT may be longer or shorter than $180 \mathrm{~ms}$, with current strengths more than 2 times threshold, basic drive cycle lengths of more than $600 \mathrm{~ms}$ or less than $400 \mathrm{~ms}$, use of isopro- terenol to facilitate induction of VT or with left ventricular stimulation. Further, because all episodes of VT and VF were induced in the absence of antiarrhythmic drug therapy, the results of this study cannot be applied to programmed stimulation during electropharmacologic testing.

None of the nonclinical VTs in this study were unimorphic. Although some forms of unimorphic VT that have never been documented may be induced in patients who have a documented history of a particular form of unimorphic VT, these forms of VT wore not included in the present study because of uncertainty as to whether they are truly nonclinical or clinical but undocumented. ${ }^{4}$

Conversely, none of the clinical VTs in this study were polymorphic VT or VF. Although polymorphic VT or VF is often the documented arrhythmia at the time of resuscitation in patients with out-of-hospital cardiac arrest, the initiating arrhythmia in such patients may frequently be unimorphic VT. ${ }^{13,14}$ Because the clinical significance of polymorphic VT or ventricular fibrillation induced by programmed stimulation in patients with a history of out-of-hospital cardiac arrest is unclear, ${ }^{6}$ such patients were excluded from this study.

Approximately $50 \%$ of the nonclinical arrhythmias included in this study were sustained and required direct-current countershock to terminate. Because nune of the patients with induced sustained polymorphic VT or VF had a history of a cardiac arrest or had received countershocks in the past, these sustained arrhythmias were clearly nonclinical in these patients. However, it may be argued that some of the nonsustained episodes of polymorphic VT induced in this study may have also occurred spontaneously, but may have been asymptomatic and undocumented. This possibility cannot be ruled out and is a limitation of the study. However, the means and distributions of the coupling intervals that induced the sustained and nonsustained forms of the nonclinical arrhythmias did not differ, and therefore the results would have been similar even if the nonclinical arrhythmias had been restricted to those that were sustained.

Acknowledgment: We are grateful to Lisa Hackbarth for her excellent secretarial assistance.

\section{References}

1. Mann DE, Luck JC, Griffín JC, Herre JM, Limacher MC, Magro SA, Robertson NW, Wyndham CRC. Induction of clinical ventricular tachycardia using programmed stimulation: value of third and fourth extrastimuli. Am J Cardinl 1983;52:501-506.

2. Brugada P, Green M, Abdollah $H$, Wellens HJJ. Significance of ventricular arrhythmias initiated by programmed ventricular stimulations: the importance of the type of ventricular arrhythmia induced and the number of premature stimuli required. Circulation 1984;69:87-92.

3. Buxton AE. Waxman HL, Marchlinski FE, Untereker WJ, Waspe LE, Josephson ME. Role of triple extrastimuli during electrophysiologic study of patients with documented sustained ventricular tachyarrhythmias. Circulation 1984;69:532-540.

4. Morady F, DiCarlo L, Winston S, Davis JC, Scheinman MM. A prospective comparison of triple extrastimuli and left ventricular stimulation in studies of ventricular tachycardia induction. Circulation 1984;70:52-57.

5. Brugada P, Wellens HI]. Comparison in the same patient of two programmed ventricular stimulation protocols to induce ventricular tachycardia. 
Am I Cardiol 1985;55:380-383

6. Wellens HJJ, Brugada P, Stevenson WG. Programmed electrical stimulation of the heart in patienls with life-threutening ventricular arrhythmias: what is the significance of induced arrhythmias and what is the correct 3timulation protecol? Girnulation 1985:72:1-7.

7. Morady F, Shapiro W. Shen E, Sung RI, Scheinman MM. Progrummed ventricular stimulation in patients without spontaneous ventricular tachycardia. Am Heart T 1984;107:875-882.

8. Brugada $\mathrm{P}$, Abdollah $\mathrm{H}$, Heddle B, Wellens $\mathrm{HJJ}$. Results of a ventricular stimulation protocol using a maximum of 4 premature stimuli in patients without documented or suspected ventricular arrhythmias. Am I Cardiol 1983;52:1214-1218.

9. Morady F, DiCarlo LA, Liem LB, Krol RB, Baerman JM. Effects of high stimulation current on the induction of ventricular tachycardia. Am J Cardiol 1985;56:73-78

10. Morady F, Shen E, Schwartz A, Hess D, Bhandari A, Sung RJ, Scheinman
MM. Long-term follow-up of patients with recurrent unexplained syncope evaluated by electrophysiologic testing. IACC 1983;2:1053-1059.

11. DiCarlo LA Jr, Morady F, Schwartz AB, Shen EN, Baerman JM, Krol RB, Scheinman MM, Sung RJ. Clinical significance of ventricular fibrillationflutter induced by ventricular programmed stimulation. Am Heart / 1985;

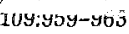

12. Doherty JU, Pcmbrook-Rogers D, Grogan EW, Falcone RA, Buxton AE, Marchlinski FE, Cassidy DM, Kienzle MC, Almendral JM, Josephson ME. Electrophysiologic evaluation and follow-up characteristics of patients will recurrent unexplained syncope and presyncope. Am I Cardiol 1985;55:703708 .

13. Kempf FC Ir, Josephson ME. Cardiac arrest recorded on ambulatory electrocardiograms. Am J Cardiol 1984:53:1577-1582.

14. Milner PG, Platia EV, Reid PR, Griffith LSC. Ambulatory electrocardiographic recordings at the time of fatal cardiae arrest. Am J Cardiol 1985; $56: 588-592$ 